\title{
Outflow Facility and Extent of Angle Closure in a Porcine Model
}

Ying Hong, ${ }^{1,2}$ Chao Wang, ${ }^{1,3,4}$ Ralitsa Loewen, ${ }^{1}$ Susannah Waxman, ${ }^{1}$ Priyal Shah, ${ }^{1}$ Hamed Esfandiari, ${ }^{1,5}$ Nils A. Loewen ${ }^{1 *}$

1: Department of Ophthalmology, University of Pittsburgh Medical Center, Pittsburgh, Pennsylvania, United States

2: Department of Ophthalmology, Peking University Third Hospital, Beijing, China

3: Department of Ophthalmology, Xiangya Hospital, Central South University, Changsha, Hunan, China.

4: The Third Xiangya Hospital of Central South University, Changsha, Hunan, China.

5: Department of Ophthalmology, Northwestern University, Chicago, Illinois, United States

Nils A. Loewen, MD, PhD

203 Lothrop St

Suite 819

Pittsburgh, PA 15213

Email: Loewen.nils@gmail.com

Fax: +001-412-605-1541 


\begin{abstract}
Purpose: To investigate the extent of anterior chamber angle circumference needed to maintain a physiological outflow facility (C).
\end{abstract}

Methods: Twenty anterior segments of porcine eyes were assigned to 5 groups, each with a different degree of cyanoacrylate-mediated angle closure: $90^{\circ}(n=4), 180^{\circ}(n=4), 270^{\circ}(n=4), 360^{\circ}(n=4)$ and four unoccluded control eyes. The outflow facility was measured at baseline, 3, 12, 24, and 36 hours after angle closure. Outflow patterns were evaluated with canalograms and the histomorphology was compared.

Results: Baseline outflow facilities of the five groups were similar ( $F=0.922, P=0.477$ ). Complete, circumferential occlusion over $360^{\circ}$ induced a significant decrease in facility from baseline at all timepoints ( $P \leq 0.023$ at $3,12,24$ and 36 hours). However, no difference from baseline was found in any of the partially-occluded $\left(0-270^{\circ}\right)$ groups ( $\mathrm{F} \geq 0.067, \mathrm{P} \geq 0.296$ at $3,12,24$ and 36 hours). The canalograms confirmed the extent of occlusion with flow through the unblocked regions. Histology revealed no adverse effects of blockage on the TM or aqueous plexus in the unoccluded angle portions. The unoccluded TM appeared normal.

Conclusion: Cyanoacrylate-mediated angle occlusion created a reproducible angle closure model. $90^{\circ}$ of unoccluded anterior chamber angle circumference was sufficient to maintain physiological outflow. This model may help understand how outflow can be regulated in healthy, nonglaucomatous TM.

Keywords: angle closure model, glaucoma, trabecular meshwork, outflow facility 


\section{Introduction}

Primary angle closure glaucoma (PACG) has a prevalence between $0.5-2.5 \%$ and is the main type of glaucoma in patients of Asian ethnicity [1, 2]. The diagnosis and treatment of PACG have been well described [3-5]. Recent studies identified several anatomic factors associated with angle closure that include a thicker iris with greater curvature, increased lens vault, and a smaller anterior chamber volume, width, and area [6-9]. However, there is limited knowledge about how much open angle area is needed to maintain a normal intraocular pressure (IOP) and how the remaining trabecular meshwork (TM) accommodates the need for increased outflow. Not only would such insight allow for development of better treatments, understanding the molecular mechanism of focal facility increase in normal eyes may provide further insight into how it fails in primary open angle glaucoma. We created a cyanoacrylate-mediated angle closure model using our porcine anterior segment perfusion culture system [10-12]. Porcine eyes share many structural, biochemical, and physiological features with human eyes and are available in sufficient quantities within a short time since procurement [10-13]. Cyanoacrylate has been used to seal corneal perforations and glaucoma filtering bleb leaks [14-16] because it adheres safely and effectively to living tissues $[17,18]$. For this reason, we used cyanoacrylate to create a standardized and accessible angle closure model by occluding the anterior chamber angle extensively. The aim of this study was to investigate the extent of anterior chamber angle circumference necessary to maintain a normal outflow facility.

\section{Methods}

\section{Angle closure model}

Twenty porcine eyes were obtained from a local abattoir (Thoma Meat Market, Saxonburg, Pittsburgh PA) within two hours of sacrifice, decontaminated via submersion in $5 \%$ povidone-iodine ophthalmic solution (Betadine 5\%, Fisher Scientific, NC9771653) for two minutes, and hemisected in an aseptic flow hood. After removal of the posterior segment, lens, and ciliary body, anterior segments were mounted on custom perfusion dishes [19-22]. They were perfused at the height equivalent of 15 $\mathrm{mmHg}$ for 30 minutes to establish a stable baseline facility. The perfusion media consisted of Dulbecco's modified Eagle's media (DMEM, Fisher Scientific, sh30284.02) supplemented with 1\% FBS (Fisher Scientific, 10082-147) and 1\% antibiotic, antimycotic (Fisher Scientific, 15240-062).

Perfusion cultures were divided into five groups with different ranges of cyanoacrylate-mediated anterior chamber angle occlusion (Elmer's Products Inc. Westerville, OH, U.S.A). The treatment groups 
consisted of occlusion of $90^{\circ}(n=4), 180^{\circ}(n=4), 270^{\circ}(n=4)$, and $360^{\circ}(n=4)$ while the control group included 4 cyanoacrylate-free eyes. Using an ophthalmic operating microscope, cyanoacrylate was carefully applied by an eye surgeon to the corresponding range of TM to ensure proper occlusion. Anterior segments were remounted in the perfusion dishes and perfused at $15 \mathrm{mmHg}$ for 36 hours at $37^{\circ} \mathrm{C}$ and $5 \% \mathrm{CO}_{2}$.

\section{Outflow facility measurement}

The volume of the outflow facility was measured at baseline, 3, 12, 24, and 36 hours after angle occlusion. The facility was calculated for each time point using the Goldmann equation $(\mathrm{C}=$ volume/time/IOP) [23].

\section{Microsphere canalograms}

Fluorescent microsphere canalograms were obtained after perfusion of two eyes in each treatment group as previously described [12]. Briefly, fluorescent microspheres (FluoSpheres, $0.5 \mu \mathrm{m}$, red fluorescent [505/515], 2\% solids; Thermo Fisher Scientific, Eugene, OR, USA) were diluted 100-fold in media and perfused at the height equivalent of $15 \mathrm{mmHg}$. Fluorescence was visualized with a stereo dissecting microscope equipped for fluorescent imaging (Olympus SZX16 with GFP filter cube and DP80 monochrome/color camera; Olympus Corp., Center Valley, PA, USA). Images were captured (CellSens; Olympus Life Science, Tokyo, Japan) using $2 \times 2$-pixel binning and a resulting resolution of $580 \times 610$ pixels.

\section{Histology}

At the end of the experiment, anterior segment was fixed in 4\% PFA for 48 hours. Samples were embedded in paraffin, and sagittal sections of the angle were stained with hematoxylin and eosin (H\&E) for histological evaluation.

\section{TM viability and TM cytoskeleton}

After 36 hours of perfusion, anterior segments were dissected and incubated with calcein AM (C1430; Thermo Fisher, Waltham, MA, USA, $0.3 \mu \mathrm{M}$ ) and propidium iodide (PI, P1304MP; Thermo Fisher, Waltham, MA, USA, $1 \mu \mathrm{g} / \mathrm{ml}$ ) for $30 \mathrm{~min}$ at $37^{\circ} \mathrm{C}[20,24]$. After three washes with phosphate-buffered saline (PBS), the TM was flat-mounted and imaged with an upright laser scanning confocal microscope at 400-fold magnification (BX61; Olympus, Tokyo, Japan). 
To visualize F-actin, the TM was fixed with 4\% PFA for 24 hours and stained with phalloidin (1:20, A12379, Thermo Scientific, Waltham, MA) for 30 minutes and DAPI (D1306, Thermo Fisher Scientific, Waltham, MA) as a nuclear stain. Images were acquired using a Fluoview FV1200 upright confocal microscope (BX61; Olympus, Tokyo, Japan) at the same magnification as above.

\section{Statistical analysis}

Outflow facility (C) of each group was reported as the mean \pm SD. Intergroup and intragroup facilities were compared using one-way analysis of variance (ANOVA). Facility differences between each treatment group were assessed with the Least Significant Difference (LSD) $t$-test. All tests were conducted with SPSS 18.0 software package (SPSS Inc., Chicago, IL, USA), and a p-value $<0.05$ was considered statistically significant.

\section{Results}

The baseline outflow facility of the five groups at baseline and 3, 12, 24 and 36 hours after angle occlusion (Table 1 ) showed no statistically significant intergroup difference $(F=0.922, P=0.477)$. Additionally, no significant differences between baseline and post-occlusion facilities post-treatment were found in eyes with up to $270^{\circ}$ of occlusion ( $F=0.067, P=0.976$ at 3 hours, $F=0.960, P=0.443$ at 12 hours, $F=1.381, P=0.296$ at 24 hours, $F=1.170, P=0.362$ at 36 hours, respectively). Complete circumferential occlusion did cause a significant decrease in facility $(P=0.020$ at 3 hours, 0.023 at 12 hours, 0.006 at 24 hours, 0.023 at 36 hours, respectively).

Fluorescent microsphere canalograms obtained after 36 hours of perfusion for each group were visible in the unoccluded angle section (Fig. 2) confirming the extent of angle closure, while the entire angle was labeled in controls. Histology revealed TM as a multilayered, porous, and sparsely pigmented tissue. Unoccluded control eyes had typical outflow tract morphology with uveal, corneoscleral, and juxtacanalicular meshwork, and an angular aqueous plexus with rudimentary, Schlemm's canal-like segments. The cyanoacrylate membrane covered the corresponding trabecular meshwork area, as shown by the transparent region marked with arrowheads (Fig. 3a). Eyes with angle occlusion had a looser appearing TM while beams and aqueous spaces were otherwise preserved (Fig. 3b). The outflow tract morphology and cellularity adjacent to the occluded angle regions were not appreciably different from those in the unoccluded controls (Fig. 3c). 
TM cells from the unoccluded control group stained positively with calcein AM (Fig. 4a). TM cells from adjacent unoccluded regions were mostly calcein AM positive while only occasionally stained with PI (Fig. 4b). In contrast, no calcein AM staining but many PI-stained cells were found in the portion once coated with cyanoacrylate (Fig. 4c). Morphological differences in the above three groups suggested that application of cyanoacrylate was not detrimental to TM cells in the non-occluded areas.

The F-actin microfilaments in unoccluded flat-mounted TM tissue samples showed weak, spotlike, or segmental staining (Fig. 5a). F-actin microfilaments in TM tissue adjacent to the occluded angle (Fig. 5b) were similar to those of the control group. This was in contrast to weak F-actin signals in the occluded portion (Fig. 5c).

\section{Discussion}

This study compared the outflow facility in anterior segments of porcine eyes with different extents of angle closure. At least $90^{\circ}$ of unoccluded angle was necessary to prevent a significant change from the baseline facility. This suggests that the remaining TM can increase flow sufficiently to maintain a physiological outflow facility without obvious detriment to the microscopic appearance of the TM, to its cell viability or cytoskeleton.

Drainage of aqueous humor outflow via the conventional pathway is IOP dependent and includes the TM, Schlemm's canal and its endothelium, the collector channels and aqueous veins [25]. There is also a pressure independent uveoscleral outflow [26-28] as well as a limited transscleral flow [29]. The minimal flow in the $360^{\circ}$ angle closure group can be attributed mostly to transscleral flow since the uvea is removed in our model. In contrast to the eye with complete angle closure, the outflow facility of eyes with $90^{\circ}, 180^{\circ}$, and $270^{\circ}$ of occlusion was not significantly different from the control. Outflow through the TM is segmental rather than uniform under physiological conditions [30-32], suggesting that only a portion of the available capacity is active at a given time [33-38]. Hann et al. found that with increasing perfusion pressure, more regions label with tracer demonstrating the TM's ability to adapt and upregulate its outflow capacity [34]. These findings are in accordance with our results. As little as $90^{\circ}$ of open angle was sufficient to maintain healthy outflow without negative effects on the unoccluded tissue.

Our experimental results and those of others $[33,39]$ match the common clinical observation of segmental pigmentation near collector channels, possibly indicating a higher flow in these areas [40]. The anterior chamber angle is usually divided into quarters of $90^{\circ}$ circumference to describe the 
classification or diagnosis of angle disorders. If the posterior TM is seen for less than $90^{\circ}$ of the angle circumference, this is deemed an occludable angle [41].

Several limitations of this study must be noted. While porcine eyes feature biochemical glaucoma markers and many physiological properties similar to those of human eyes [42], their angular aqueous plexus is different, with a thicker TM and limbus-parallel canal segments rather than a Schlemm's canal $[43,44]$. Additionally, low cytotoxic effects of 2-octyl-cyanoacrylate have been described $[15,45]$. However, cyanoacrylate has been used for over half a century in a variety of surgical applications, including wound closure [46], tissue grafting [47], and treatment of gastric varices [48], without clinically relevant toxicity-related complications at the tissue level. In the eye, it can be used to seal corneal perforations, lesions, bleb leaks, and secure tube shunts, among other applications $[14,17,18]$.

In summary, we created a porcine angle closure model with an adjustable angle closure range. We found that $90^{\circ}$ of open angle circumference was sufficient to maintain the outflow facility within a normal range.

\section{Funding}

This study was funded by the Initiative to Cure Glaucoma of the Eye and Ear Foundation of Pittsburgh (NAL), by NEI Grant K08EY022737 (NAL), by NIH CORE Grant P30 EY08098 to the Department of Ophthalmology, and an unrestricted grant from Research to Prevent Blindness, New York, NY.

\section{Compliance with ethical standards}

Conflict of Interest The authors declare no conflict of interest.

Ethical approval This article does not contain any studies with human participants or animals performed by any of the authors. No animals were sacrificed for the purpose of doing research. An approval by an ethics committee or institutional animal care and use committee were not required. 


\section{References}

1. Liang Y, Friedman DS, Zhou Q, et al (2011) Prevalence and Characteristics of Primary Angle-Closure Diseases in a Rural Adult Chinese Population: The Handan Eye Study. Invest Ophthalmol Vis Sci 52:8672-8679

2. Casson RJ, Newland HS, Muecke J, et al (2007) Prevalence of glaucoma in rural Myanmar: the Meiktila Eye Study. Br J Ophthalmol 91:710-714

3. Wright C, Tawfik MA, Waisbourd M, Katz LJ (2016) Primary angle-closure glaucoma: an update. Acta Ophthalmol 94:217-225

4. Chen S, Lv J, Fan S, et al (2017) Laser peripheral iridotomy versus laser peripheral iridotomy plus laser peripheral iridoplasty in the treatment of multi-mechanism angle closure: study protocol for a randomized controlled trial. Trials 18:130

5. Foster PJ, Buhrmann R, Quigley HA, Johnson GJ (2002) The definition and classification of glaucoma in prevalence surveys. Br J Ophthalmol 86:238-242

6. Nongpiur ME, Ku JYF, Aung T (2011) Angle closure glaucoma: a mechanistic review. Curr Opin Ophthalmol 22:96-101

7. Nongpiur ME, Sakata LM, Friedman DS, et al (2010) Novel association of smaller anterior chamber width with angle closure in Singaporeans. Ophthalmology 117:1967-1973

8. Wang B-S, Narayanaswamy A, Amerasinghe N, et al (2011) Increased iris thickness and association with primary angle closure glaucoma. Br J Ophthalmol 95:46-50

9. Nongpiur ME, He M, Amerasinghe $\mathrm{N}$, et al (2011) Lens vault, thickness, and position in Chinese subjects with angle closure. Ophthalmology 118:474-479

10. Loewen RT, Brown EN, Roy P, et al (2016) Regionally Discrete Aqueous Humor Outflow Quantification Using Fluorescein Canalograms. PLoS One 11:e0151754

11. Loewen RT, Roy P, Park DB, et al (2016) A Porcine Anterior Segment Perfusion and Transduction Model With Direct Visualization of the Trabecular Meshwork. Invest Ophthalmol Vis Sci 57:13381344

12. Loewen RT, Brown EN, Scott G, et al (2016) Quantification of Focal Outflow Enhancement Using Differential Canalograms. Invest Ophthalmol Vis Sci 57:2831-2838

13. Bachmann B, Birke $M$, Kook D, et al (2006) Ultrastructural and biochemical evaluation of the porcine anterior chamber perfusion model. Invest Ophthalmol Vis Sci 47:2011-2020

14. Okabe M, Kitagawa K, Yoshida T, et al (2013) Application of 2-octyl-cyanoacrylate for corneal perforation and glaucoma filtering bleb leak. Clin Ophthalmol 7:649-653

15. Vote BJ, Elder MJ (2000) Cyanoacrylate glue for corneal perforations: a description of a surgical technique and a review of the literature. Clin Experiment Ophthalmol 28:437-442 
16. Kusabara AA, Kasahara N (2017) Managing glaucoma drainage device tube leak with cyanoacrylate. Acta Ophthalmol 95:e662

17. Guhan S, Peng S-L, Janbatian H, et al (2018) Surgical adhesives in ophthalmology: history and current trends. Br J Ophthalmol. doi: 10.1136/bjophthalmol-2017-311643

18. García-Delpech S, Sanz-Marco E, Martinez-Castillo S, et al (2013) Ahmed valve, suture-less implantation: A new approach to an easier technique. J Glaucoma 22:750-756

19. Loewen RT, Roy P, Park DB, et al (2016) A Porcine Anterior Segment Perfusion and Transduction Model With Direct Visualization of the Trabecular Meshwork. Invest Ophthalmol Vis Sci 57:13381344

20. Dang Y, Waxman S, Wang C, et al (2017) Freeze-thaw decellularization of the trabecular meshwork in an ex vivo eye perfusion model. PeerJ 5:e3629

21. Dang Y, Loewen R, Parikh HA, et al (2017) Gene transfer to the outflow tract. Exp Eye Res 158:73-84

22. Loewen RT, Brown EN, Roy P, et al (2016) Regionally Discrete Aqueous Humor Outflow Quantification Using Fluorescein Canalograms. PLoS One 11:e0151754

23. Brubaker RF (2004) Goldmann's equation and clinical measures of aqueous dynamics. Exp Eye Res 78:633-637

24. Gonzalez JM Jr, Hamm-Alvarez S, Tan JCH (2013) Analyzing live cellularity in the human trabecular meshwork. Invest Ophthalmol Vis Sci 54:1039-1047

25. Tamm ER (2009) The trabecular meshwork outflow pathways: structural and functional aspects. Exp Eye Res 88:648-655

26. Karlova E, Zolotaryov A, Nikolayeva G (2009) Trabecular meshwork contribution to the uveoscleral outflow. Acta Ophthalmol 87:0-0

27. Toris CB, Gregerson DS, Pederson JE (1987) Uveoscleral outflow using different-sized fluorescent tracers in normal and inflamed eyes. Exp Eye Res 45:525-532

28. Toris CB, Yablonski ME, Wang YL, Camras CB (1999) Aqueous humor dynamics in the aging human eye. Am J Ophthalmol 127:407-412

29. Kleinstein RN, Fatt I (1977) Pressure dependency of transcleral flow. Exp Eye Res 24:335-340

30. Keller KE, Bradley JM, Vranka JA, Acott TS (2011) Segmental versican expression in the trabecular meshwork and involvement in outflow facility. Invest Ophthalmol Vis Sci 52:5049-5057

31. Cha EDK, Xu J, Gong L, Gong H (2016) Variations in active outflow along the trabecular outflow pathway. Exp Eye Res 146:354-360

32. Yang C-YC, Liu Y, Lu Z, et al (2013) Effects of Y27632 on aqueous humor outflow facility with changes in hydrodynamic pattern and morphology in human eyes. Invest Ophthalmol Vis Sci 54:5859-5870 
33. Swaminathan SS, Oh D-J, Kang MH, Rhee DJ (2014) Aqueous outflow: segmental and distal flow. J Cataract Refract Surg 40:1263-1272

34. Hann CR, Bahler CK, Johnson DH (2005) Cationic ferritin and segmental flow through the trabecular meshwork. Invest Ophthalmol Vis Sci 46:1-7

35. Chang JYH, Folz SJ, Laryea SN, Overby DR (2014) Multi-scale analysis of segmental outflow patterns in human trabecular meshwork with changing intraocular pressure. J Ocul Pharmacol Ther 30:213223

36. Stamer WD, Acott TS (2012) Current understanding of conventional outflow dysfunction in glaucoma. Curr Opin Ophthalmol 23:135-143

37. Carreon T, van der Merwe E, Fellman RL, et al (2016) Aqueous outflow-A continuum from trabecular meshwork to episcleral veins. Prog Retin Eye Res S1350-9462

38. Goel M, Picciani RG, Lee RK, Bhattacharya SK (2010) Aqueous humor dynamics: a review. Open Ophthalmol J 4:52-59

39. Hann CR, Fautsch MP (2009) Preferential fluid flow in the human trabecular meshwork near collector channels. Invest Ophthalmol Vis Sci 50:1692-1697

40. Johnson DH (1989) Does pigmentation affect the trabecular meshwork? Arch Ophthalmol 107:250254

41. Foster PJ, Aung T, Nolan WP, et al (2004) Defining "occludable" angles in population surveys: drainage angle width, peripheral anterior synechiae, and glaucomatous optic neuropathy in east Asian people. Br J Ophthalmol 88:486-490

42. McMenamin PG, Steptoe RJ (1991) Normal anatomy of the aqueous humour outflow system in the domestic pig eye. J Anat 178:65-77

43. Suarez T, Vecino E (2006) Expression of endothelial leukocyte adhesion molecule 1 in the aqueous outflow pathway of porcine eyes with induced glaucoma. Mol Vis 12:1467-1472

44. Tripathi RC (1971) Ultrastructure of the exit pathway of the aqueous in lower mammals: (A preliminary report on the "angular aqueous plexus"). Exp Eye Res 12:311-314

45. Trott AT (1997) Cyanoacrylate tissue adhesives. An advance in wound care. JAMA 277:1559-1560

46. Habib A, Mehanna A, Medra A (2013) Cyanoacrylate: a handy tissue glue in maxillofacial surgery: our experience in alexandria, egypt. J Maxillofac Oral Surg 12:243-247

47. Adler N, Nachumovsky S, Meshulam-Derazon S, Ad-El D (2007) Skin graft fixation with cyanoacrylate tissue adhesive in burn patients. Burns 33:803

48. Kozieł S, Kobryń K, Paluszkiewicz R, et al (2015) Endoscopic treatment of gastric varices bleeding with the use of n-butyl-2 cyanoacrylate. Prz Gastroenterol 10:239-243 


\section{Legends}

Figure 1

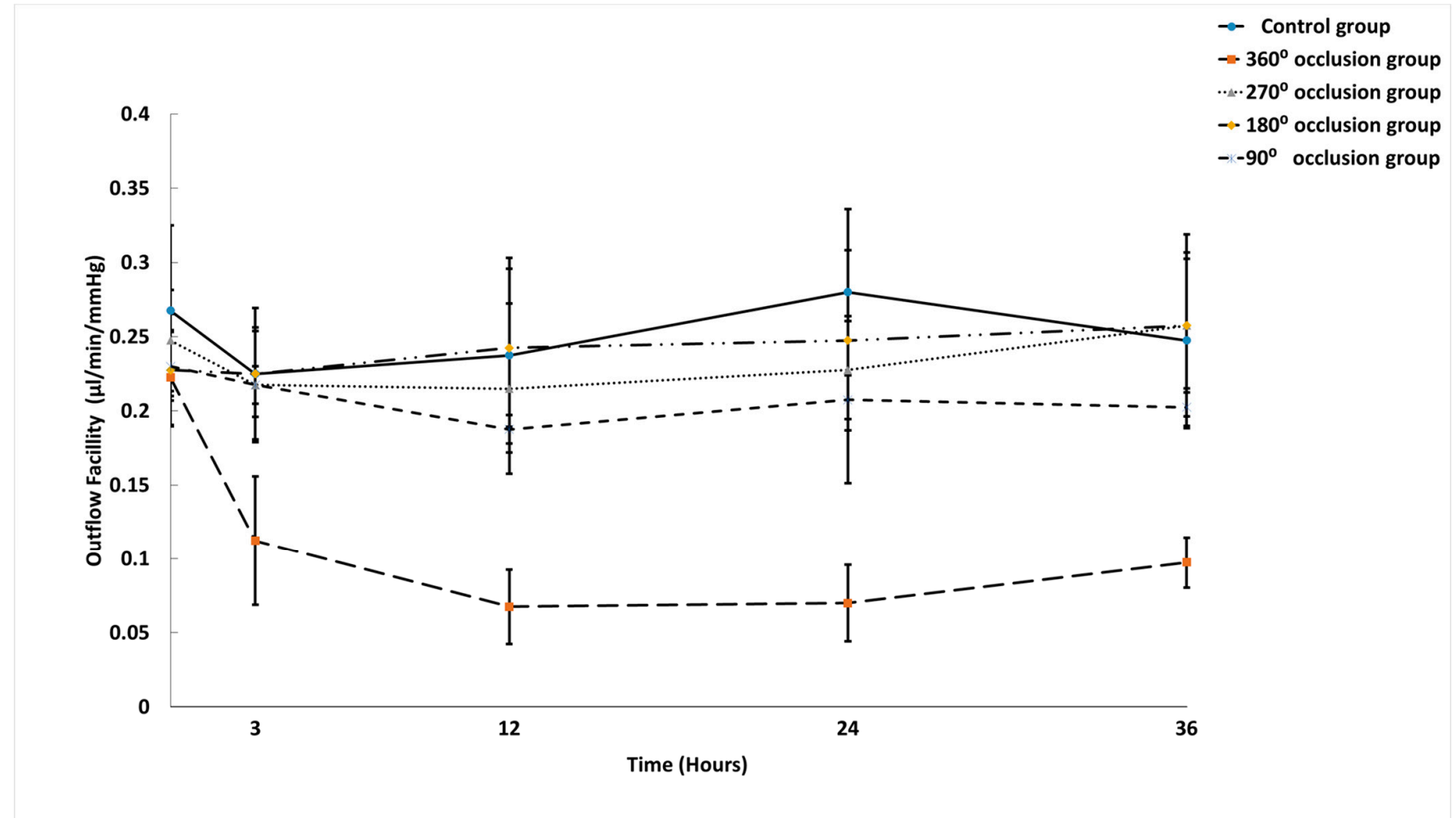

Fig. 1: Outflow facility of all groups. Only the $360^{\circ}$ angle closure group had a significantly decreased facility. 


\section{Figure 2}
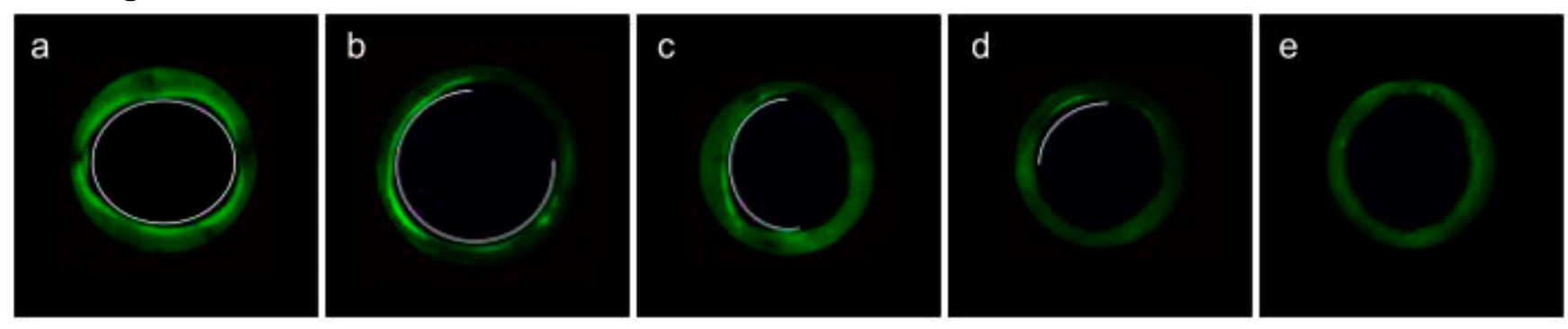

Fig. 2: Representative canalograms of experimental groups. (a) Control eyes with fluorescent spheres throughout circumference. (b) $90^{\circ}$ angle closure group had fluorescent spheres throughout the unoccluded angular aqueous plexus. (c) $180^{\circ}$ angle closure group with corresponding fluorescence. (d) $270^{\circ}$ angle closure group with fluorescent spheres in the remaining quadrant. (e) $360^{\circ}$ angle closure group. 


\section{Figure 3}
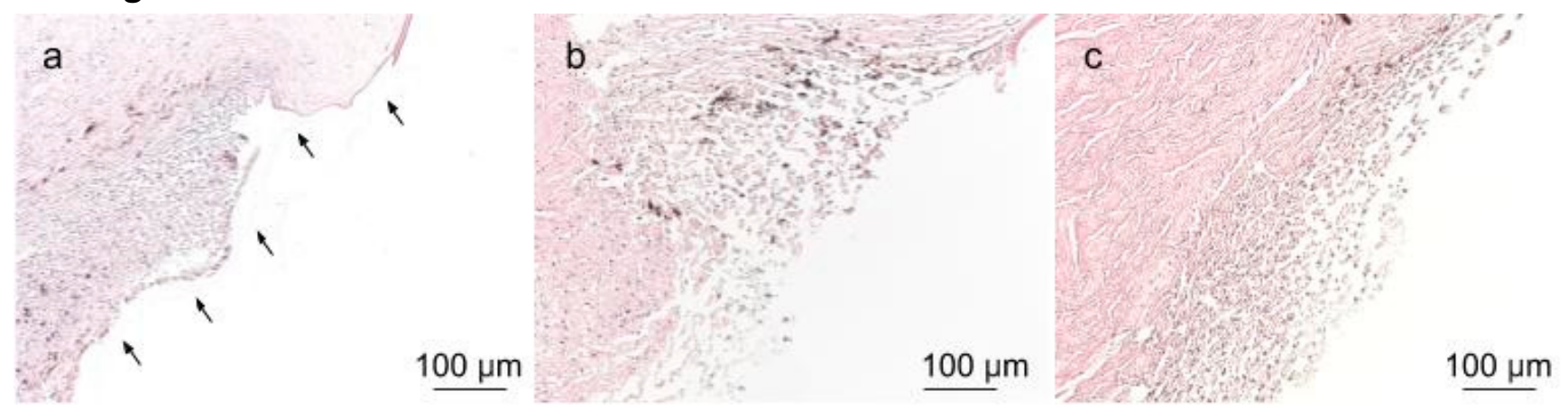

Fig. 3. Comparative anterior chamber angle histology in the anterior chamber model. (a) The cyanoacrylate membrane covered the corresponding trabecular meshwork area(arrowheads). Angular aqueous plexus section with angle closure appeared loose with reduced TM cell count. (b) Non-occluded portion of eye with cyanoacrylate-mediated angle closure had an appearance similar to the control eyes. (c) Control eye with porcine angular aqueous plexus. 


\section{Figure 4}
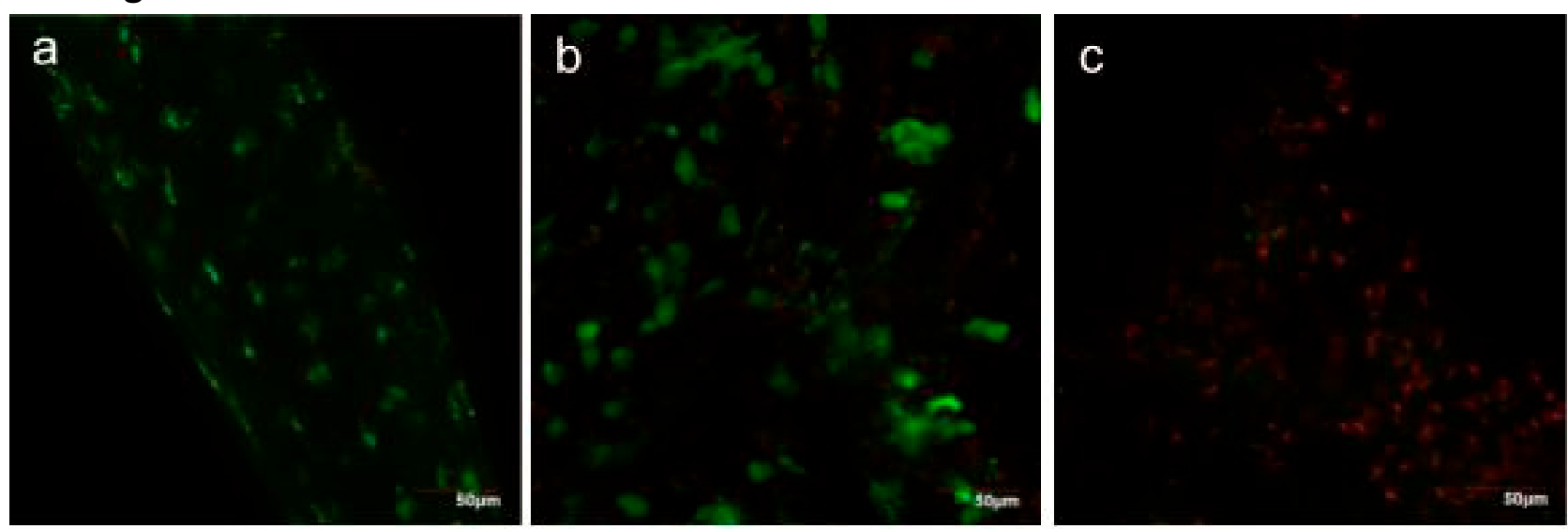

Fig. 4: Immunofluorescent staining for calcein AM (green) and propidium iodide (red) in TM cells from various groups. (a) TM cells in the control group readily stained with calcein AM. (b) Cells from nonoccluded TM directly adjacent to areas with angle closure showed similar staining patterns to the control (calcein AM positive) with occasional PI-stained cells. (c) TM cells from eyes with complete angle closure showed rare calcein AM staining and positive results for PI staining. 


\section{Figure 5}
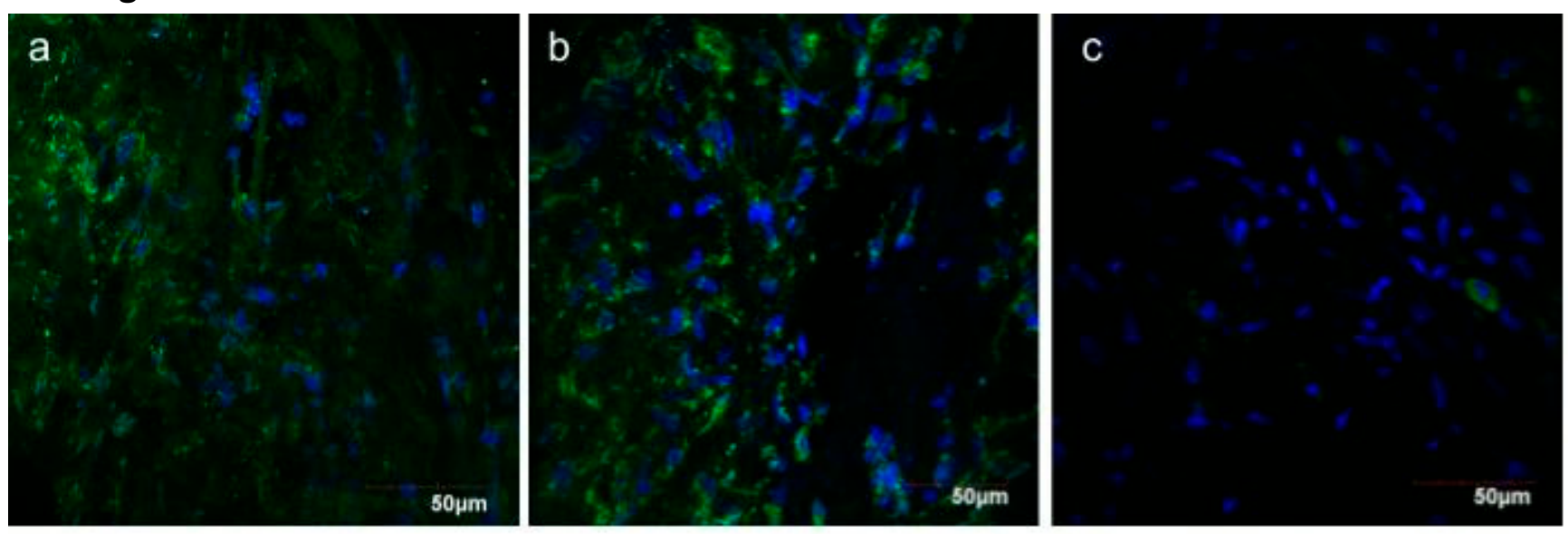

Fig. 5: Immunofluorescent staining for F-actin (green) and nuclear staining (blue) in TM cells from

different groups. (a) TM cells from the control group showed positive F-actin microfilament staining. (b) Cells from the non-glued TM portion of the eye with angle closure showed similar staining patterns to the control. (c) Weak F-actin staining resulted in TM cells of the occluded portion of the eye with angle closure. 